\title{
L'intrigue du gouverneur, le gouvernement d'une intrigante? Mme de Genlis et Mme de Merteuil dans les Liaisons Dangereuses de Laclos
}

\author{
Lisanne Jansen ${ }^{1}$ (D)
}

Published online: 9 March 2017

(C) The Author(s) 2017. This article is published with open access at Springerlink.com

\begin{abstract}
The two eighteenth-century novelists Choderlos de Laclos and Mme de Genlis were rivals in different fields. They were both employed by the duke of Orléans and thus occupied a public function. They both wrote an epistolary novel in 1782 and they both had very outspoken opinions about the widely debated subject of female education. The main hypothesis ventured in this article is that Laclos has used Mme de Genlis as a model for the immoral character of Mme de Mertueil in his famous novel Les Liaisons Dangereuses. Mme de Genlis' contemporaries have mentioned a possible link between her and the character of Mme de Merteuil, but they did not mention which character traits the countess of Genlis could possibly have in common with this character. The above-mentioned, three-folded rivalry between Laclos and Mme de Genlis will serve as a starting point in order to understand why Mme de Genlis' contemporaries could have made a link between her and Laclos' character.
\end{abstract}

Keywords Genlis · Laclos · Rivalry · Theater · Education · Politics

Après la parution des Liaisons Dangereuses de Choderlos de Laclos en mars 1782, les lecteurs du roman cherchent des pistes concernant les personnages qu'avait peints le général de Laclos. Le sens de l'intrigue de M. de Valmont a été mis en rapport avec l'esprit conspirateur que devait posséder Laclos lui-même en tant qu'acteur de la Révolution française au service du duc d'Orléans (Garat 1862, 430). Une autre source importante des personnages constitue la société grenobloise où

Lisanne Jansen

L.Jansen@student.ru.nl

1 Radboud University, Nijmegen, The Netherlands 
Laclos séjournait avec son régiment d'artillerie entre 1769 et 1775 , c'est-à-dire avant sa carrière politique au Palais-Royal. Les clefs grenobloises se réduisent principalement à deux textes que presque tous les historiens ont inlassablement cités: les confidences de Laclos au comte de Tilly dans ses Mémoires et l'œuvre autobiographique La Vie de Henri Brulard de Stendhal. En ce qui concerne Mme de Merteuil, la complice rusée de Valmont, on a ainsi proposé une certaine marquise de L.T.D.P.M et une Mme de Montmaur comme des modèles ayant pu inspirer Laclos (Versini 1968, 26). Tilly et Stendhal ne disent quasiment rien sur les ressemblances concrètes entre Mme de Merteuil et les deux Grenobloises. La marquise de L.T.D.P.M possède «des traits dignes des jours des impératrices romaines» (Gontaut et Tilly 1862, 317-318) et quant à Mme de Montmaur on apprend seulement que le jeune Stendhal a vu «cette fin de mœurs» (Stendhal 1982, 593) chez elle.

Une deuxième source d'inspiration importante est la société parisienne que fréquentait Laclos (Versini 1968, 32). Ainsi, dans sa biographie de Félicité Stéphanie, comtesse de Genlis, Gabriel de Broglie écrit qu'à la parution du roman, le bruit courait dans les salons que Mme de Merteuil partageait des traits avec la comtesse de Genlis (Broglie 1985, 168). Mme de Genlis était connue à l'époque pour le ton moralisateur et édifiant de son œuvre ainsi que pour son rôle de gouvernante, puis de gouverneur ${ }^{1}$ des enfants du duc d'Orléans. La question se pose de savoir quels auraient pu être ces traits que la comtesse pouvait partager avec une intrigante hypocrite, dont Mme Riccoboni, autre femme des lettres de cette époque, disait qu'elle possédait un «caractère affreux» (Riccoboni et Laclos 1979, 760).

Une rivalité entre Laclos et Mme de Genlis dans trois domaines intrinsèquement liés, à savoir, la littérature, l'éducation et la politique fournit des indices qui permettraient d'identifier des ressemblances concrètes entre le personnage des Liaisons et la comtesse. Il ne s'agira pas ici d'avancer l'hypothèse selon laquelle Mme de Genlis serait l'archétype unique de Mme de Merteuil, thèse qui chercherait à remplacer les autres modèles proposés. Comme l'explique Laclos lui-même dans sa correspondance avec Mme Riccoboni au sujet de l'existence de Mme de Merteuil: le Tartuffe de Molière est une accumulation de l'hypocrisie de nombreuses personnes, et ainsi les «noirceurs» et «l'hypocrisie des mœurs» de nombreuses femmes ont été réunies dans le caractère de Mme de Merteuil (Riccoboni et Laclos 1979, 761). Ce que nous proposons donc ici, c'est de s'interroger sur le personnage de la marquise à partir de la rivalité entre deux proches du duc d'Orléans à la veille de la révolution française. De cette façon nous voudrions éclaircir pourquoi les contemporains de Laclos ont pu remarquer des ressemblances entre le personnage des Liaisons Dangereuses et Mme de Genlis.

\footnotetext{
${ }^{1}$ Le poste de gouverneur, qui était responsable de l'éducation des enfants après qu'ils avaient été gardés par leur gouvernante, était réservé aux hommes d'épee. La nomination de Mme de Genlis était pour cette raison une chose extraordinaire.
} 


\section{La querelle littéraire: mimer l'exemplarité morale}

Dans sa biographie du général de Laclos, Emile Dard fait remarquer que celui-ci triomphait avec ses Liaisons Dangereuses dans «ce genre délicat, dont madame de Genlis s'attribuait orgueilleusement le monopole» (Dard 1905, 43). La comtesse avait publié son propre roman épistolaire Adèle et Théodore en janvier 1782. En termes de vente de roman, Mme de Genlis bat Laclos dans les années 1780 et 1790 (Cook 1991, 371-383). Au niveau du contenu, les similarités, mais surtout les différences entre les deux œuvres sont frappantes. Mme de Genlis et Laclos dépeignent la corruption morale de la société de l'Ancien Régime. Tandis que Mme de Genlis présente la religion et l'éducation comme des moyens qui peuvent faire ressortir l'homme de cette décadence morale, une telle solution semble absente dans l'œuvre de Laclos (Dow 2007, xvii). Ajoutons que le désir d'exemplarité morale ressortant du roman de Mme de Genlis contraste avec les idées sur l'éducation morale de Laclos. Le lieu de départ de ce dernier aspect de la rivalité littéraire entre les deux personnes se situe antérieurement à la publication d'Adèle et Théodore et réside dans les idées contrastantes de Laclos et Mme de Genlis sur la mise en scène de la vertu et de la méchanceté au théâtre.

La correspondance entre Mme Riccoboni et Laclos au sujet de la moralité des Liaisons est très illustrative de l'affinité avec le théâtre de ce dernier ainsi que de son désir de mettre en scène le méchant. Mme Riccoboni reproche à l'auteur des Liaisons l'invraisemblance des caractères affreux qu'il a peints. Dans sa réponse, Laclos réplique qu'il ne pense pas que les caractères monstrueux de Valmont et de Merteuil soient irréels, étant de l'opinion qu'ils existent bel et bien mais de manière cachée et qu'il a «répandu l'alarme et dévoilé leurs coupables artifices» (Riccoboni et Laclos 1979, 760). La peinture du méchant a pour fonction de le dénoncer. Laclos reprend de cette manière un procédé théâtral rendu célèbre par Molière. Laurent Versini souligne la présence du Tartuffe de Molière et ses avatars du XVIIIe siècle chez Gresset, Desmahis et le marquis de la Noue chez Laclos. «Le nouveau Tartuffe», écrit-il «est le Tartuffe des mœurs [...] Valmont lui aussi joue et met en scène un nouveau Tartuffe, celui de la bienfaisance» (Versini 1968, 69). Ce Tartuffe parodie ou dégrade le modèle de bienfaisance de l'honnête homme bourgeois promu par Diderot et d'autres dans le drame bourgeois (Ibidem). Ainsi, dans la lettre XXI, Valmont raconte qu'afin de soigner sa réputation, il a secouru une famille qui ne pouvait payer ses dettes, se comparant au héros d'un drame (Laclos 2011, 55-57). De cette manière, il veut conquérir la prude Mme de Tourvel dont il est tombé amoureux. Cependant, Valmont a obtenu ce conseil - c'est-à-dire de couvrir sa réputation par un masque vertueux - de Mme de Merteuil dans la lettre X. La question se pose alors de savoir de quelles sources théâtrales ${ }^{2}$ l'instructrice de Valmont s'est inspirée afin de transmettre ses leçons à son élève libertin.

Laclos parodie dans les Liaisons le voile de la bienséance morale des femmes aristocrates de son temps (Pomeau 1975, 151-152). La personne de Mme de Genlis ne constituerait-elle dans ce cas qu'un exemple parmi tant d'autres candidates?

\footnotetext{
${ }^{2}$ Voir pour les sources romanesques dont le personnage de Mme de Merteuil se serait inspirée l'ouvrage de Christine Roulston (Roulston 1998).
} 
Certes, la préoccupation morale et le désir d'austérité de la comtesse contrastait avec le style de vie mondaine de sa carrière aristocrate et pour cette raison elle se trouve accusée d'inauthenticité (Robb 2008, 16). Ajoutons qu'elle a entretenu une relation amoureuse secrète avec le duc de Chartres, le futur duc d'Orléans pour qui elle a occupé le rôle de gouverneur de ses enfants (Harmand 1912, 110). Toutefois, même si cette opinion explique pourquoi ses contemporains ont pu dresser intuitivement une analogie entre le caractère de la comtesse et celui de l'hypocrite Mme de Merteuil, elle ne précise pas pourquoi la comtesse de Genlis spécifiquement a pu inspirer Laclos pour l'hypocrisie morale de Mme de Merteuil. La prédilection pour le théâtre de Mme de Genlis, ainsi que les idées concernant la nécessité de la mise en scène théâtrale de l'exemplarité vertueuse qui traversent son œuvre théâtrale pour les enfants, pourraient éclaircir ce point.

Dès son enfance, Mme de Genlis a joué du théâtre et il paraît qu'elle avait beaucoup de talent (Broglie 1985, 49-55). Pourtant ce talent lui rapportait aussi la réputation dubitable d'être factice et de ne jouer qu'un rôle théâtral dans la vie réelle (Robb 2008, 40-44). Mme de Genlis semble un personnage paradoxal dont la vie et l'œuvre se sont dissociées à l'âge adulte (Broglie 1985, 60-61). Elle était une des figures principales du théâtre d'éducation, un genre destiné aux enfants qui nouait quant à son désir d'exemplarité moral avec le drame bourgeois (Plagnol-Diéval 1997, 38-40). Le théâtre d'éducation se trouve, comme le formule si bien MarieEmmanuelle Plagnol-Diéval «à mi-chemin entre littérature et réalité» (PlagnolDiéval 1997, 10), facilitant de cette manière la mise en pratique des leçons transmises par la fiction. Dans la préface de l'éditeur du Théâtre à l'usage des jeunes personnes (1780), que Mme de Genlis avait déjà publié avant Adèle et Théodore et qui sert de toile de fond au roman épistolaire, elle écrit: «en jouant ces Pièces, en les apprenant par cœur, elles [les jeunes personnes] y trouveront plusieurs avantages, ceux de graver dans leur souvenir des principes excellents» (Genlis 1780, 1). Par la voie du théâtre, les exemples fictifs deviennent plus réels. Il faut de bons exemples afin de les imiter, et il faut éviter de mettre en scène le méchant. Ainsi, Mme de Genlis évite consciencieusement le procédé moliéresque célébré par Laclos: peindre le vice pour le dénoncer.

Dans Adèle et Théodore, le désir de peindre les bons exemples est repris. Le roman commence par une lettre du baron d'Almane au vicomte de Limours, dans laquelle ce premier lui fait savoir qu'il a déménagé avec sa femme et ses deux enfants Adèle et Théodore en Languedoc. Il veut dérober «l'enfance de [s]on fils aux exemples du vice» (Genlis 1782, 2). C'est pour cette raison, que la baronne d'Almane fait jouer du théâtre à ses enfants. Pourtant elle est critique pour ce qui est des pièces qu'elle recommande: la baronne d'Almane décourage explicitement l'usage de l'œuvre de Molière parce que selon elle «la morale n'est jamais qu'un accessoire et non le but principal» (Genlis 1782, 263) dans les pièces du grand dramaturge du XVII ${ }^{\mathrm{e}}$ siècle. De plus, elle ne fait lire son œuvre à sa fille Adèleprobablement pour cette raison—qu'à l'âge de dix-huit ans (Ibidem).

Si nous devons croire Laclos, une telle exemplarité morale n'est qu'un jeu d'apparences qui embrume la tête de la jeunesse des mensonges agréables de ce décor vertueux. Son personnage Mme de Merteuil est friande du théâtre et d'autres spectacles. Au fil du roman, elle invite la jeune Cécile de Volanges plusieurs fois à 
des sorties à l'Opéra. De plus, elle confie à Valmont que le théâtre prend une place considérable dans sa propre formation. Dans les conseils qu'elle donne à ce dernier résonne une connaissance du théâtre d'éducation. Mme de Merteuil avertit que Mme de Tourvel a «rendu les illusions de la jeunesse» et des «heureuses témérités» (Laclos 2011, 34) à Valmont. Celui-ci est devenu admirateur de la vertu enfantine de cette dame. Ces douces illusions rappellent les principes vertueux que les enfants doivent apprendre par cœur pendant leurs leçons de théâtre recommandées par Mme de Genlis ainsi que la crainte d'un mauvais comportement que l'on leur y inspire. Tandis que Valmont tourne en dérision le drame bourgeois, Mme de Merteuil s'attaque donc au genre théâtral qui a voulu transmettre l'exemplarité bourgeoise à la jeunesse. Mme de Merteuil fait remarquer ironiquement que Valmont, prenant sans doute son rôle de héros de drame un peu trop au sérieux, se conduit maintenant sans principes. Cela veut dire qu'il semble avoir oublié comment il peut détourner la vertu en ruse pour faire réussir sa conquête amoureuse. Mme de Merteuil l'estime suffisamment mûr pour se laisser dégoûter de la vertu. Dans ce but, elle lui donne l'exemple de sa conquête d'un certain Chevalier en étant «tour à tour enfant et raisonnable, folâtre et sensible, quelquefois même libertine» (Laclos 2011, 37-38). De nouveau on retrouve l'image de l'enfant innocent qui a assidûment appris par cœur des principes vertueux, pour les abuser une fois devenu adulte.

Mme de Merteuil ne donne pas seulement des conseils à Valmont. Le triomphe de ses instructions se trouve dans le rôle de pédagogue qu'elle assure auprès de la jeune Cécile de Volanges et son amant Danceny. Cela nous amène au deuxième domaine de rivalité entre Mme de Genlis et Laclos: l'éducation, et particulièrement l'éducation féminine à l'aide du théâtre.

\section{La querelle éducative: une Mme de Merteuil pédagogue}

La rivalité littéraire entre Mme de Genlis et Laclos se trouve étroitement liée aux idées contrastantes qu'ils possèdent sur l'éducation. Depuis 1777, Mme de Genlis est la gouvernante des filles du duc d'Orléans et en 1782 elle devient également le gouverneur de ces fils, parmi lesquels le futur roi Louis-Philippe. En 1788, Laclos s'installe également au Palais-Royal au service du duc d'Orléans. La nouvelle de l'arrivée de Laclos au Palais-Royal déplaisait profondément à Mme de Genlis et ce n'est qu'à cette époque qu'elle avoue avoir lu son œuvre, qu'elle traite d' «exécrable par les principes» (Genlis 1825, 11) dans ses Mémoires. Considérée la réputation infâme du livre, il était devenu un scandale d'en avouer la lecture. ${ }^{3}$ L'arrivée de Laclos a rompu ce silence de la part de Mme de Genlis et elle fait usage de sa connaissance du livre pour faire savoir au duc qu'elle abandonnera immédiatement l'éducation de ses enfants s'il consent à donner la place prévue de secrétaire des commandements à l'auteur des Liaisons (Genlis 1825, 10). Mme de Genlis n'a pas tenu sa parole, mais sa réaction virulente trahit déjà l'intime rapport de sa rivalité

\footnotetext{
3 Catriona Seth, dans la préface de l'édition Pléiade la plus récente des Liaisons dit que «[1]e refus de lire - ou d'avouer qu'on a lu - le livre devient topique» citant à ce sujet en particulier la comtesse d'Holstein et Mme de Genlis.
} 
avec Laclos sur le plan littéraire avec leurs idées sur l'éducation. Le mauvais exemple littéraire que constituait le roman de Laclos allait à l'encontre de son plan d'éducation pour les enfants du duc, basé sur l'exemplarité morale. Ce n'est pour autant pas le seul point de friction entre elle et Laclos dans le domaine éducatif.

Laclos s'oppose à l'idée selon laquelle les princes ou d'autres enfants auraient besoin d'une gouvernante ou d'une éducatrice autre que leur propre mère. Il reprend de cette manière une idée qui gagnait beaucoup de terrain dans la deuxième moitié du XVIII siècle, sous l'influence de notamment Rousseau : la nature féminine exige qu'une femme renonce à la sphère publique pour assurer une fonction morale en tant que mère de ses enfants (Steinbrügge 1995, 6). Ainsi, dans un essai qui fait partie d'une trilogie réunie sous le nom De l'Education des femmes, Laclos pose une question rhétorique moqueuse :«lequel du fils d'un prince ou de cet enfant sauvage, lequel des deux est abandonné en naissant?» (Laclos 1979, 395). Laclos se montre donc de l'avis que l'éducation que reçoivent les enfants d'une autre personne que leur mère, témoigne de négligence. Pourtant, le point central que fait Laclos dans ces trois essais est que la femme éduquée spécifiquement constitue un danger pour la société et pour elle-même.

Jean Bloch fait remarquer que Mme de Genlis est du moins partiellement d'accord avec Laclos sur ce dernier point. Dans Adèle et Théodore Mme de Genlis explique qu'il est dangereux et inutile pour une femme de posséder le génie et le goût des études, même si elle est de l'opinion que les jeunes filles ont droit à une meilleure éducation que celle qu'elles ne recevaient d'ordinaire à l'époque. Les connaissances que doivent acquérir les femmes se limitent à celles qui leur sont nécessaires pour gérer une maison et élever leurs enfants (Bloch 1984, 145-146). Pour illustrer cette idée Mme de Genlis se sert d'une référence à la pièce les Femmes savantes de Molière (Genlis 1782, 460). Dans cette pièce, il est question de femmes qui étalent leurs connaissances d'une façon pédante. Le type de connaissances dont les femmes disposent idéalement selon Mme de Genlis ne frise pas la pédanterie. Elles reçoivent une formation austère et pratique qui vise la maternité, idéal, comme le fait remarquer Bloch, qu'elle semble partager avec Laclos.

Toutefois, dans Polixène, un texte court qui fait partie de la Galérie des dames françaises $^{4}$ et qui a été attribué à Laclos par Versini comme un «portrait-charge de son ennemi Mme de Genlis» (Laclos, Versini 1979, 1572), Laclos traite les idées de la comtesse-Polixène de «médiocres». La difficulté réside ici dans le fait que Laclos qualifie l'austérité de Mme de Genlis-Polixène d'hypocrite. Polixène «revêtit un caractère d'austérité, qui souleva les prudes, en imposa aux sots, amusa les connaisseurs, et surprit ceux qui n'ont pas le temps d'examiner» (Laclos, Versini 1979, 749-751). La note de Versini concernant cette phrase est très intéressante: «c'est la tactique de Mme de Merteuil» (Ibidem, 1572), écrit-il, dressant de cette manière implicitement un lien triangulaire entre Polixène, le personnage des Liaisons et Mme de Genlis.

Comme Polixène, Mme de Merteuil impose ses idées aux naïfs, c'est-à-dire à ceux qui sont susceptibles de reprendre tout bêtement ce qu'elle dit. Reprenant un

\footnotetext{
${ }^{4}$ Une collection de 40 portraits de dames françaises distinguées.
} 
vers de la comédie Le Méchant ${ }^{5}$ de Gresset, elle dit à Valmont que «[1]es sots sont ici-bas pour nos menus plaisirs» (Laclos 2011, 798). La fonction qu'elle occupe pour la naïve Cécile de Volanges est celle d'une institutrice qui remplace sa mère. Dans la lettre XXIX, nous apprenons par Cécile qu'elle est heureuse «qu'une femme qui [lui] est presque pas parente, prenne plus de soin d'[elle] que [s]a mère!» (Ibidem, 124). Au fil du roman, cette relation se révèle de plus en plus nocive pour le caractère de Cécile: Mme de Merteuil se révèle une véritable liaison dangereuse pour la jeune fille (Versini 1968, 155). Elle commence à éduquer Cécile à l'aide des romans sentimentaux qu'elle lui prête. La lecture de ces romans doit enseigner la fille à mieux écrire et à bien se conduire. Cependant, le but final de cette éducation est de lui apprendre comment elle peut masquer sa relation amoureuse secrète avec son professeur de chant, Danceny, liaison qui rappelle l'amour impossible entre Julie et son précepteur Saint-Preux dans la Nouvelle Héloïse de Rousseau. L'exemple vertueux imité par la jeune Cécile et son amant sert d'astuce pour couvrir une intention adultère. Il faut cependant, pour reprendre une des maximes de Mme de Genlis, des leçons de théâtre pour mettre en pratique ces «principes excellents».

Le lieu d'apprentissage pour ces leçons théâtrales est très révélateur. Rappelons que sous l'influence de Rousseau, l'idéal d'une éducation naturelle gagnait du terrain dans la deuxième moitié du XVIIIe siècle. Le lieu d'apprentissage devait refléter cet idéal et son Emile, comme exemple de tous les enfants, doit être élevé à la campagne (Rousseau 1966, 112). Mme de Genlis ne faisait pas exception à la règle. Elle élève les enfants du duc à Bellechasse, une résidence à la campagne, loin du Palais-Royal, où elle tient aussi salon et accueille beaucoup de gens de lettres, des familiers de la maison d'Orléans et d'autres aristocrates (Broglie 1985, 98-100; 175-179). A Bellechasse elle fait jouer son théâtre d'éducation par ses élèves: la nature et l'artifice se joignent dans ce lieu. Son roman épistolaire Adèle et Théodore, qui porte pour sous-titre Lettres sur l'éducation contenant tous les principes relatifs à l'éducation des Princes, des jeunes personnes et des hommes, reflète sur ce point et d'autres l'éducation des enfants du duc. Mme de Genlis décrit dans ce roman l'éducation des enfants d'une certaine baronne d'Almane, qui rappelle quant à ces principes éducatifs rigoureux Mme de Genlis elle-même. La baronne a déménagé avec son mari et ses enfants au Languedoc, loin de la ville tumultueuse de Paris où elle joue avec eux des pièces de son Théâtre à l'usage des enfans \& des jeunes personnes, œuvre qui rappelle évidemment le Théâtre à l'usage des jeunes personnes écrit par Mme de Genlis elle-même (Genlis 1782, 210-211).

La campagne comme lieu pour donner des leçons par le moyen du théâtre est repris chez Laclos. La fuite en province d'Adèle et Théodore rencontre son double dans les Liaisons. Pour faire réussir son plan adultère, Mme de Merteuil veut que Cécile et Danceny partent ensemble à la campagne. De plus, elle insiste sur le fait que les enfants sont des acteurs pour qui ce lieu spécial offre des opportunités. Dans la lettre LXIII, Valmont, le complice du projet de Mme de Merteuil, est chargé sur

\footnotetext{
${ }^{5}$ Madame de Genlis a écrit une comédie qui a pour titre Le Méchant par Air qui résonne dans cette lettre. Dans cette pièce Mme de Genlis fait usage de l'expression «le danger des liaisons» attesté déjà avant Laclos.
} 
demande de cette dernière de réunir les deux élèves amoureux loin de leur domicile parisien.

C'est de vos soins que va dépendre le dénouement de cette intrigue. Jugez du moment où il faudra réunir les Acteurs. La Campagne offre mille moyens; et Danceny à coup sûr, sera prêt à s'y rendre à votre premier signal. Une nuit, un déguisement, une fenêtre que sais-je, moi ? Mais enfin, si la petite fille en revient telle qu'elle y aura été, je m'en prendrai à vous. Si vous jugez qu'elle ait besoin de quelque encouragement de ma part, mandez-le-moi. Je crois lui avoir donné une assez bonne leçon sur le danger de garder des Lettres, pour oser lui écrire à présent; et je suis toujours dans le dessein d'en faire mon élève (Laclos 2011, 154).

Le lieu d'apprentissage que constitue la campagne où les «Acteurs» se réunissent rappelle les leçons de théâtre de Mme de Genlis à Bellechasse et le rôle d'éducatrice que veut assumer Mme de Merteuil pour Cécile rappelle le rôle que Mme de Genlis assurait pour les enfants du duc d'Orléans. Il faudrait ajouter maintenant que Mme de Merteuil adopte sa fonction d'éducatrice dans le but d'influencer la société qu'elle fréquente. Autrement dit, l'intrigue de son théâtre en tant que gouvernante se transforme en un gouvernement d'intrigante habile. En établissant la relation entre Cécile et Danceny, elle veut se venger de Gercourt, un homme qui l'a offensée et qui est désigné comme le futur mari de Cécile. Mme de Genlis était, elle aussi, soupçonnée d'avoir une influence politique en tant qu'éducatrice des enfants du duc d'Orléans, et elle avait pour concurrent aussi dans ce domaine Choderlos de Laclos.

\section{La rivalité politique: le gouvernement de Mme de Merteuil}

Parce que Mme de Genlis était une proche de la figure politique importante qu'était le duc d'Orléans, elle était soupçonnée d'occuper une position qui lui donnerait de l'influence politique à la veille de la Révolution (Harmand 1912, 116-117). Avec l'arrivée de Laclos au service du duc, elle doit désormais partager cette position avec l'homme qu'elle devrait haïr presque autant pour l'immoralité de son œuvre que pour ses idées sur l'éducation féminine (Birkett 1988, 150). Au XIXe siècle, Jules Michelet écrivait à ce sujet: «Regardons à ces fenêtres [du Palais-Royal]. J'y vois distinctement une femme blanche, un homme noir. Ce sont les conseillers du prince, le vice et la vertu, Mme de Genlis et Choderlos de Laclos» (Michelet 1847, 75-76). Michelet se range, du point de vue moral, clairement et de manière peu nuancée du côté de Mme de Genlis. Ce qui est d'autant plus intéressant est qu'il attribue à celle-ci en tant que gouverneur une fonction politique comparable à celle de Laclos, à une époque où il n'était pas évident pour une femme, même si elle était noble, d'occuper ouvertement une fonction politique. Dans la deuxième moitié du XVIIIe siècle la femme se retire de la sphère publique pour occuper une fonction 
morale dans la sphère privée (Lee 1975; Blanc 2006; Steinbrügge 1995). ${ }^{6}$ Cette rivalité politique est profondément ancrée dans les idées divergentes sur l'éducation féminine des deux proches du duc. En effet, Laclos et Mme de Genlis ont en commun le fait que leurs idées concernant l'éducation (féminine) sont étroitement liées à leurs idées politiques, tandis que leurs opinions divergent pour ce qui est de l'effet politique concret de cette éducation. Madame de Genlis semble considérer que la situation politique peut s'améliorer par la voie de l'éducation (féminine) tandis que Laclos est de l'opinion que l'éducation féminine constitue un danger pour la société.

En commentant le passage sur la société fictive de Lagaraye dans le deuxième tome d'Adèle et Théodore, Carolina Armenteros propose que le projet pédagogique de Madame de Genlis a un but politique: seules les personnes bien éduquées peuvent servir le bien commun. De plus, elle souligne encore une fois l'importance de l'éducation féminine dans l'œuvre de Mme de Genlis (Armenteros 2013, 47). Pendant son séjour à Lagaraye, le baron d'Almane échange des lettres ${ }^{7}$ avec le comte de Roseville au sujet de l'éducation des femmes et de leur capacité de régner. Il dit qu'il est parfaitement d'accord avec les idées du baron concernant l'éducation féminine: les mères sont parfois plus aptes que les pères ou que le plus habile instituteur quand il s'agit d'élever leurs enfants. Leur éducation, dont elles doivent transmettre les fruits à leurs enfants, est pour cette raison très importante (Genlis 1782, 169). Pourtant cette éducation diffère de celle que reçoivent les princes. Ainsi, la femme du futur roi, même si elle est intelligente, ne doit pas se mêler à la politique et celui-ci doit veiller à ce qu'elle ne s'y mêle pas.

II est donc important que le prince soit décidé d'avance à ne point se laisser gouverner par elle. Au reste, je ne prétends point inspirer à mon élève du mépris pour les femmes en général, mais je veux qu'il sache s'en défier, \& qu'il soit convaincu d'une vérité dont je suis persuadé moi-même, c'est qu'on doit toujours les tenir éloignées des grandes affaires: elles peuvent nous égaler par la raison, mais bien rarement par la prudence (Genlis 1782, 170-171).

L'éducation féminine n'a pas pour conséquence une société dominée par les femmes chez Mme de Genlis. Pour Laclos, par contre, les deux sont intrinsèquement liées. Mme de Merteuil, qui n'a pas reçu une bonne éducation, décide de s'éduquer elle-même et elle devient ainsi un danger pour les hommes et pour la société (Bloch 1984, 148). Quelle est cette formation politique qu'elle s'est donnée et qu'elle transmet à Cécile et Danceny? Et quels parallèles avec Mme de Genlis pouvons-nous distinguer?

Dans la lettre LXXXI du roman, Mme de Merteuil raconte sa jeunesse et sa formation: «Je n'avais pas quinze ans, je possédais déjà les talents auxquels la plus grande partie de nos politiques doivent leur réputation» (Laclos 2011, 171). Quels sont ces talents? «[Il] suffisait de joindre à l'esprit d'un Auteur, le talent d'un

\footnotetext{
${ }^{6}$ Ces chercheurs critiquent le pouvoir publique qu'attribuent les frères Goncourt à la femme au XVIIIe siècle: «La femme, au dix-huitième siècle, est le principe qui gouverne, la raison qui dirige, la voix qui Commande. D'un bout à l'autre du siècle le gouvernement de la femme est le seul gouvernement visible et sensible».

7 Les réponses du baron ne font pas partie du roman.
} 
Comédien [...] mais au lieu de rechercher les vains applaudissements du Théâtre, je résolus d'employer à mon bonheur ce que tant d'autres sacrifiaient à la vanité» (Laclos 2011, 173-174). Christine Roulston remarque que Mme de Merteuil a appris, en imitant le langage de la littérature sentimentale, comment elle peut prétendre être une femme vertueuse qui montre sa nature authentique tandis que sa vertu n'est que le masque d'un comportement social (Roulston 1998, 142). Cependant, il faut également du talent pour le théâtre pour mettre ce langage en pratique et jouir effectivement de l'éloquence d'un grand homme politique sans occuper pourtant cette fonction ouvertement. Sous prétexte d'occuper une fonction pédagogique qui s'accorde mieux avec sa nature féminine, Mme de Merteuil assure son influence dans la société qu'elle fréquente.

Quant à Mme de Genlis, elle a toujours nié s'être mêlée dans les affaires du duc ou dans des affaires politiques quelconques (Genlis 1825, 86). Pourtant, comme d'autres contemporains de la comtesse, Laclos, a pu tirer une conclusion différente, utilisant l'hypocrisie de la comtesse comme exemple en peignant sa Mme de Merteuil. Le comportement théâtral que doivent adopter Cécile et Danceny, doit aider à déculpabiliser leur institutrice, qui pourra de cette manière déployer son influence publique sans encombre. Afin de se déculpabiliser lui aussi, Valmont suggère cette idée à Mme de Merteuil.

Puisque vous commencez à faire des éducations, apprenez à vos élèves à ne pas rougir et se déconcerter à la moindre plaisanterie: à ne pas nier si vivement, pour une seule femme, les mêmes choses dont ils se défendent avec tant de mollesse pour toutes les autres. Apprenez-leur encore à savoir entendre l'éloge de leur Maîtresse, sans se croire obligés d'en faire les honneurs; et si vous leur permettez de vous regarder dans le cercle, qu'ils sachent au moins auparavant déguiser ce regard de possession si facile à reconnaître, et qu'ils confondent si maladroitement avec celui de l'amour. Alors vous pourrez les faire paraître dans vos exercices publics, sans que leur conduite fasse tort à leur sage institutrice et moi-même, trop heureux de concourir à votre célébrité, je vous promets de faire et de publier les programmes de ce nouveau collège (Laclos 2011, 345).

Le «déguisement de regard» que doivent maitriser Cécile et Danceny renvoie à leurs leçons de théâtre, nuisibles pour la société. Dans Des Femmes et de leur éducation Laclos avertit explicitement de l'effet abusif que peut avoir le théâtre pour la société. Faisant allusion à la Lettre sur les spectacles de Rousseau, il écrit que sous l'influence du théâtre «les regards sont devenus menteurs et perfides» (Laclos 1979,434). Les yeux des citoyens ne sont plus les limpides miroirs de leurs âmes, permettant de juger convenablement de leurs actes. Au lieu de cela, leurs regards sont devenus le prétexte troublant de leurs mauvaises intentions.

Les traits de Mme de Genlis qu'ont pu inspirer Laclos dans son portrait de Mme de Merteuil se résument donc de la manière suivante. D'abord il y a la prédilection pour le théâtre, l'artifice et le style de vie aristocratique de la comtesse qui contrastent avec sa prédilection pour l'exemplarité morale en apparence. Il faut ajouter à cela son rôle d'éducatrice d'enfants qui n'étaient pas les siens à l'aide du théâtre. Et finalement, il faut mentionner l'influence politique que ce rôle 
d'éducatrice pouvait lui rapporter. C'est probablement leur coïncidence dans un même personnage, ainsi que la rivalité réelle entre Laclos lui-même et la comtesse dans les domaines littéraire, éducatif et politique, qui rendent vraisemblable l'idée selon laquelle Mme de Genlis aurait été un des étalons concrets qui ont inspiré Laclos pour son personnage de Mme de Merteuil.

Open Access This article is distributed under the terms of the Creative Commons Attribution 4.0 International License (http://creativecommons.org/licenses/by/4.0/), which permits unrestricted use, distribution, and reproduction in any medium, provided you give appropriate credit to the original author(s) and the source, provide a link to the Creative Commons license, and indicate if changes were made.

\section{Références bibliographiques}

Armenteros, C. (2013). The political thought of Madame de Genlis: Rousseau's Royalist Legacy. Relief 7(1). https://www.revue-relief.org/index.php/relief/article/view/849.

Birkett, J. (1988). Mme de Genlis: The new men and the old cave. French Studies, 42, 150-164.

Blanc, O. (2006). Cercles politiques et salons du début de la Révolution (1789-1793). Annales historiques de la Révolution française, 344, 63-92.

Bloch, J. (1984). Laclos and women's education. French Studies, 38(2), 144-158.

Cook, M. (1991). Adèle et Théodore ou les Liaisons dangereuses. Studies on Voltaire \& the Eighteenth Century, 284, 371-383.

Dard, E. (1905). Le Général Choderlos de Laclos, auteur des Liaisons Dangereuses. Paris: Perrin.

de Broglie, G. (1985). Madame de Genlis. Paris: Perrin.

Dow, G. (2007). Introduction. En G. Dow (Éd.),Mme de Genlis, Adélaide et Théodore. Chawton House Library series, Londres et New York: Routledge.

Edmond et Jules, G. (1862a). La Femme au dix-huitième siècle. Paris: Didot frères.

Garat, D. J. (1862). De la conspiration d'Orléans. En A.-L. Gontaut et A. de Tilly (Éds.), Mémoires du duc de Lauzun et du comte de Tilly. Paris: Firmin Didot frères.

Genlis, F. S, comtesse de. (1780). Théâtre à l'usage des jeunes gens. tome I. Paris: J.E Dufour \& P.H Roux.

Genlis, F. S, comtesse de. (1782). Adèle et Théodore ou Lettres sur l'éducation contenant tous les principes relatifs à l'éducation des Princes, des jeunes personnes et des hommes. Paris: M. Lambert \& F.J Baudouin.

Genlis, F. S, comtesse de. (1825). Mémoires inédits de Mme la Comtesse de Genlis, sur le dix-huitième siècle et la Révolution française, depuis 1756 jusqu'à nos jours. tome IV. Paris: Lavocat.

Gontaut, A.-L., \& de Tilly, A. (1862). Mémoires du duc de Lauzun et du comte de Tilly. Paris: Firmin Didot.

Edmond et Jules, G. (1862b). La Femme au dix-huitième siècle. Paris: Didot frères.

Harmand, J. (1912). Madame de Genlis. Sa vie intime et Politique (1746-1830). Paris: Perrin.

Laclos, C. (2011). Les Liaisons Dangereuses, éd. Catriona Seth. Paris: Gallimard (Bibliothèque de la Pléiade).

Laclos, C. (1979). Des femmes et de leur éducation. éd. Laurent Versini. Paris: Gallimard (Bibliothèque de la Pléiade).

Laclos, C. (1979). Polixène, éd. Laurent Versini. Paris: Gallimard (Bibliothèque de la Pléiade).

Laclos, Choderlos de, et Mme de Riccoboni. (1979). Correspondance entre Mme Riccoboni et M. Laclos, éd. Laurent Versini. Paris: Bibliothèque de la Pléiade.

Lee, V. (1975). The reign of women. Massachussetts: Schenkman Publishing Company.

Michelet, J. (1847). Histoire de la révolution française. Paris: Chamerot.

Plagnol-Diéval, M.-E. (1997). Madame de Genlis et le théâtre d'éducation au XVIIIe siècle. Oxford: The Alden Press.

Pomeau, R. (1975). Laclos. Paris: Hatier.

Robb, B. A. (2008). Motherhood in the margins. Newark: University of Delaware Press.

Roulston, C. (1998). Virtue, gender, and the authentic self in eighteenth-century fiction. Gainesville: The University Press of Florida.

Rousseau, J.-J. (1966). Emile, éd. Michel Launay. Paris: Garnier-Flammarion. 
Russo, E. (2007). Styles of Enlightenment. Baltimore: The Johns Hopkins University Press.

Steinbrügge, L. (1995). The moral sex. Woman's nature in the French Enlightenment. New York: Oxford University Press.

Stendhal. (1982). Vie de Henri Brulard. éd. V. Del Litto. Paris: Gallimard (Bibliothèque de la Pléiade). Versini, L. (1968). Laclos et la Tradition. Paris: Klincksieck. 